\title{
Radio resource management for public femtocell networks
}

\author{
Yizhe Li*, Zhiyong Feng, Shi Chen, Yami Chen, Ding Xu, Ping Zhang and Qixun Zhang
}

\begin{abstract}
With evolution and popularity of radio access technologies, the radio resource is becoming scarce. However, with fast-growing service demands, the future advanced wireless communication systems are expected to provide ubiquitous mobile broadband coverage to support higher data rate. Therefore, it is becoming an important problem that how to meet the greater demand with limited resources? In this situation, the femtocell has recently gained considerable attention. It is an emerging wireless access point that can improve indoor coverage as well as reduce bandwidth load in the macrocell network, and seem to be more attracted since the indoor traffic is up to $75 \%$ of all in $4 \mathrm{G}$ network. According to the newest researches, deployment of femtocell base station in public places' applications (campus, enterprise, etc.) is of much broad prospect, which could provide high quality, high rate wireless services to multiple users as well as effectively improved resource utility. However, a key challenge of the public femtocell networks is the utility-based resource management. In public femtocell networks, multi-units are necessary to jointly provide high rate, high quality services to indoor users, but there is often heavy resource competition as well as mutual interference between multiple femtocells. Therefore, it's very critical to optimize the radio resources allocation to meet femtocells' requirement as possible and reduce interference. What is more, using some ingenious resource allocation technique, multiple femtocells can cooperate and improve the system performance further. In this article, we proposed a systematic way to optimize the resource allocation for public femtocell networks, including three schemes of different stages: (1) long-term resource management, which is to allocate spectrum resource between macrocell and femtocell networks; (2) medium-term resource management, which is to allocate radio resources to each femtocell; (3) fast resource management, which is to further enable multiple femtocells to cooperate to improve the network's coverage and capacity. Numerical results show that these radio resource management schemes can effectively improve radio resource utility and system performance of the whole network.
\end{abstract}

Keywords: radio resource management, public femtocell networks, resource utility, system performance

\section{Introduction}

With evolution and popularity of radio access technologies, the radio resource is becoming scarce. However, with fast-growing service demands, the future advanced wireless communication systems are expected to provide ubiquitous mobile broadband coverage to support higher data rate. Therefore, it is becoming an important problem that how to meet the greater demand with limited resources? In this situation, the femtocell has recently gained considerable attention. It is an emerging

\footnotetext{
*Correspondence: liyizhewti@gmail.com

Wireless Technology Innovation Institutes (WTI), Key Laboratory of Universal Wireless Communications, Beijing University of Posts and

Telecommunications, Ministry of Education, No. 10 Xitucheng Road, P.O. Box 92\#, Haidian District, Beijing 100876, China
}

low-power, low-cost data access point that can improve indoor coverage as well as reduce bandwidth load in the macrocell network [1], and seem to be more attracted since the indoor traffic is up to $75 \%$ of all in $4 \mathrm{G}$ network [2]. Although femtocells were initially targeted at consumer offers, it was immediately clear that this technology presents a number of benefits for the enterprise case and for the coverage of open spaces.

According to the newest researches [3], deployment of femtocell base station (FBS) in public places' applications (campus, enterprise, etc.) are of much broad prospect, which could provide high quality, high rate wireless services to multiple users as well as effectively improved resource utility. Small office/home office

\section{苜 Springer}


business users can have immediate benefits by utilizing a consumer unit, typically with local access enabled to connect to local LAN servers. Medium and large enterprises need a different solution as multiple units need to cooperate to provide the necessary coverage and capacity. Compared to picocells the public femtocells present obvious advantages in that they do not need dedicated links.

However, a key challenge of the public femtocell networks is the utility-based resource management. Considering the large number of FBSs and the requirement of multiple units' cooperation [4], it may be high-cost and inefficient to manually allocate resource for each FBS. What is more, in public places the path loss between femtocells are weak, so the interferences between femtocells are relatively serious and the common macrocell resource management schemes may not solve this problem well. Owing to the predicted widely adoption of femtocells, researchers have begun to consider the problem of coverage optimization [5-8]. However, all of these studies focus on single femtocell coverage optimization for small-area residential users and provide good indoor coverage, preventing signals from leaking outdoors [6] as well as to increase the flexibility in deployment locations $[7,8]$, rather than on multiple femtocells that achieve joint coverage in large enterprise environments. For multiple femtocells, the main optimization goal is to optimize the resource allocation between femtocells and reduce coverage overlaps and gaps, as well as to balance the workload among femtocells.

In this article, we proposed a systematic way to optimize the resource allocation for public femtocell networks, including three schemes of different stages: (1) long-term resource management, which is to allocate spectrum resource between macrocell and femtocell networks. We proposed an adapted soft frequency reuse (ASFR) approach to combat traditional inter-cell interference by inheriting the conventional soft frequency reuse (SFR) functionality and to mitigate inter-tier interference (ITI) of macro/femtocells by applying an orthogonal spectrum reuse between macro/femtocells. In addition, we make the femtocells dynamically access macrocell's spectrum through cognitive radio (CR) technology, without interference with macrocell UEs; (2) medium-term resource management, which is to allocate radio resources to each femtocell. In this stage, we used a Q-learning-based self-configuration scheme to configure the FBS's power and work channel according to the environment in public femtocell networks. The numerical results show that the proposed scheme performed well in improving network performance as well as complexity comparing with some other common approaches; (3) fast resource management, which is to fast manage radio resources between femtocells. We proposed a coordinated multipoint transmission technique to enable femtocells to cooperate to improve the network's coverage and capacity.

The remainder of this article is organized as follows: the system model will be described in Section 2. The details and analysis of the proposed schemes will be presented in Section 3, some analytical results and performance evaluation is given in Section 4, and the last section concludes the article.

\section{System model}

Consider OFDMA-based macrocells whose frequency reuse factor $\zeta>1$, public femtocell networks (enterprise femtocells, airport femtocells, etc.) and residential femtocells are deployed in the macrocell's coverage, as shown in Figure 1. According to the traditional SFR scheme, the macrocells are partitioned into two parts: central part and outer part, femtocells may be in either central or outer part. To mitigate interference between inter-macrocells, the outer part of a cell can only use fractional spectrum and spectrums of different macrocells' outer parts are orthogonal.

Figure 2 shows a plan of the enterprise femtocell network in Figure 1. The aim of enterprise femtocell network is to meet the increasing demands for higher speed and higher-quality wireless data services within office buildings, factories, apartment buildings, and other indoor propagation environments, where the usual macrocell system can only provide degraded services or provide no coverage at all. In this article, we considered the proposed radio resource management schemes in a typical enterprise office scenario, in which there are two meeting rooms, five open offices, one demo room, and one sitting room. To provide highquality services, each room is equipped with a FBS and totally $M$ femtocells and $N$ user equipments (UEs) are distributed in this network. There is also a femto gateway which connects the FBSs with core network, collects and stores the information from all the FBSs and UEs, and allocates radio resources (power, channel etc.) between the FBSs as well as sends parameter adjustment prompt to the FBSs according to the predefined scheme.

The femtocells deployed in enterprise and campus environment usually use hybrid access mode, by which the subscribers can preferentially access the femtocells network and non-subscribers can access only when there are excess resources. The femtocells cover open places, such as railway stations, airports, and shopping malls are very similar with the enterprise ones, but for open spaces only open access mode is ever used. The discussion of access mode is beyond the scope of this article, so we consider the UEs can access the femtocell network if only there are enough resources. 


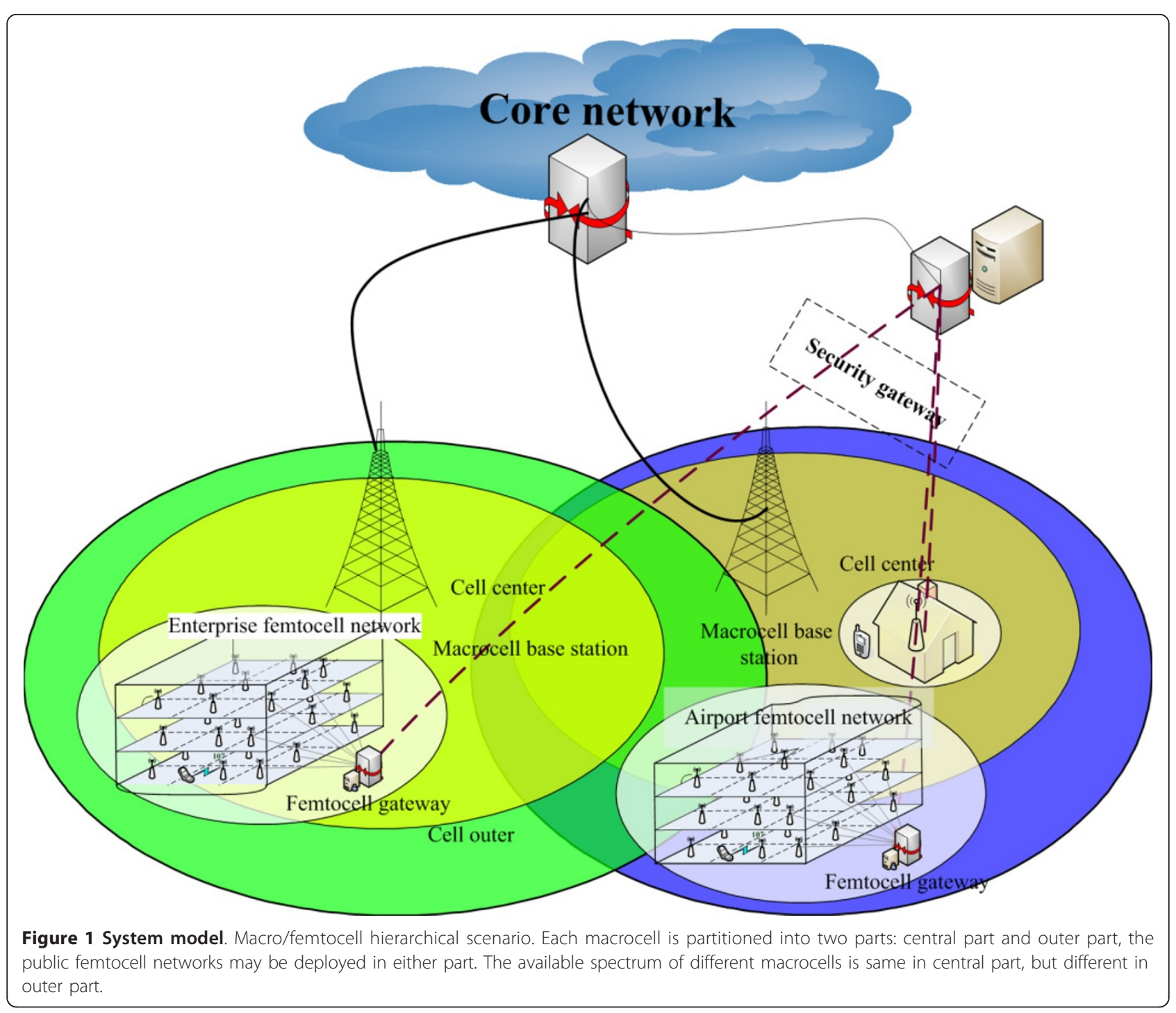

\section{Radio resource management schemes}

\subsection{Long-term resource management: spectrum} allocation between macrocell and public femtocells

Spectrum is one of the most important resources for wireless networks, public femtocell networks are no exception. Usually, there are typically two types of spectrum assignment schemes for coexistence of macrocells and femtocells [9]. One is shared spectrum allocation (co-channel), by which femtocell uses the same frequency band as the macrocell, this results in more effective use of resources and efficient hand-off (due to easier cell-search), but the interference from the macrocell BS may seriously degrade the performance. The other is split spectrum allocation, by which the femtocells use different frequency bands than those employed by the macrocell; while this avoids interference to/from the macrocell, additional spectrum resources are required.
In this article, we proposed an ASFR approach based on the traditional SFR, to eliminate interference between macrocell and femtocells, as well as maximize available spectrum resources for femtocell networks.

\subsubsection{ASFR premier}

Figure 3 gives us an overview of the ASFR spectrum allocation approach. Cells A, B, and C denote the three cells of a typical cluster. Like traditional SFR, the total available spectrum is divided into three orthogonal segments with equal size, respectively, utilized by celledge users of the three cells. Thus, the cell-edge bands of neighboring cells are orthogonal. As premier ASFR design, let $\mathrm{FBS}_{1, n}$ represent the $n$th femtocell access point within the coverage of macro base station (MBS) $A$. Assuming that the cell-edge UEs of MBS $A$ are restricted to utilize spectrum segment $j(j \in\{1,2,3\})$, then the available spectrum segments for the $\mathrm{FBS}_{1, n}$ is (a) segment $j(j \in\{1,2,3\})$, if the FBS is at the cell 


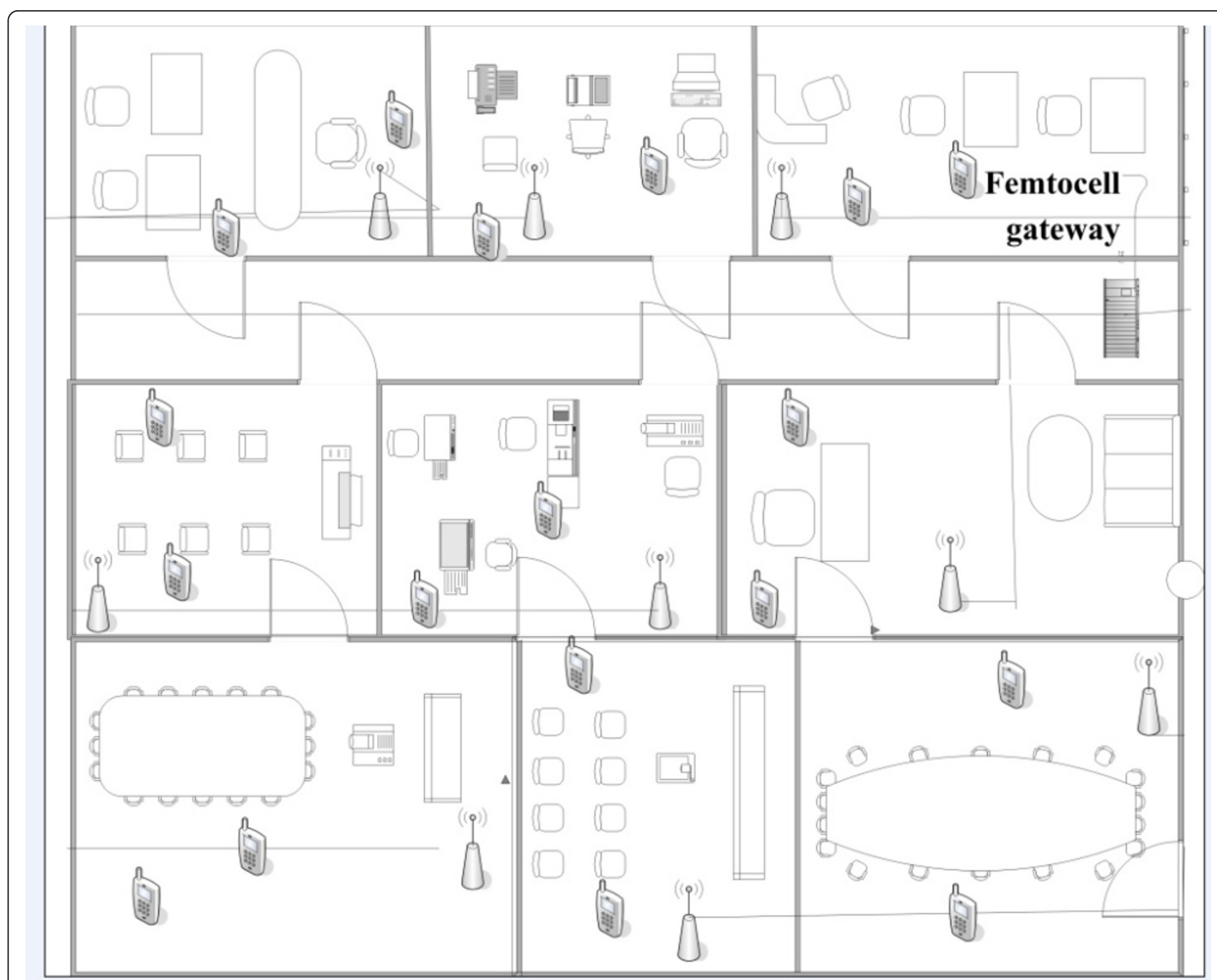

Figure 2 Enterprise femtocell network. A typical enterprise office scenario, in which there are two meeting rooms, five open offices, one demo room and one sitting room. To provide high quality services, each room is equipped with a multi-element antenna femtocell. All the femtocells are controlled by the femtocell gateway.

center; (b) the other two segments, i.e., segment $(j$ $(\bmod 3)+1)$ and segment $((j+1)(\bmod 3)+1)$, otherwise. In this way, FBSs reuse the spectrum of MBSs in an orthogonal approach and the inter-tier interference is mitigated. We should notice that the cell-center borderline $\rho$, which is the distance from the local MBS and which divides the cell-center and cell-outer users, can be tuned for performance optimization. However, the ratio of available resource numbers for cell center and cell outer is fixed at 2:1. As a result, the available number ratio of cell-center and cell-outer FBSs is fixed at 1:2, which means that the cell-center FBSs' capacity may be cut to only half of that in the cell edge, and which is unreasonable. We call this disadvantage as 1:2 issues. Therefore, to make the ASFR approach more applicable, designs to overcome this imbalance are essential.

\subsubsection{ASFR evolution}

The key ambition of this ASFR evolution is to provide designs immune to the 1:2 issue. Inspired by the EFFR approach introduced in literature [10], CR techniques is implemented in the ASFR design, and FBSs are offered additional secondary spectrum-the local macrocell radio channels, while their original available spectrum is made primary, as in Figure 3. Let $\mathrm{FBS}_{1, n}$ be in the cell edge of Cell $\mathrm{A}$, by now it has one primary spectrum segment: $j$ $(j \in\{1,2,3\})$, and two secondary spectrum segments: segment $(j(\bmod 3)+1)$ and segment $((j+1)(\bmod 3)+$ $1)$. As we all know, secondary spectrum reuse is always accompanied by sorts of spectrum detection tools and criterions, and extra costs as well. To improve spectrum efficiency, while not to make the existing cellular system become too complex, detection of the secondary spectrum is triggered if and only if the primary spectrum is 


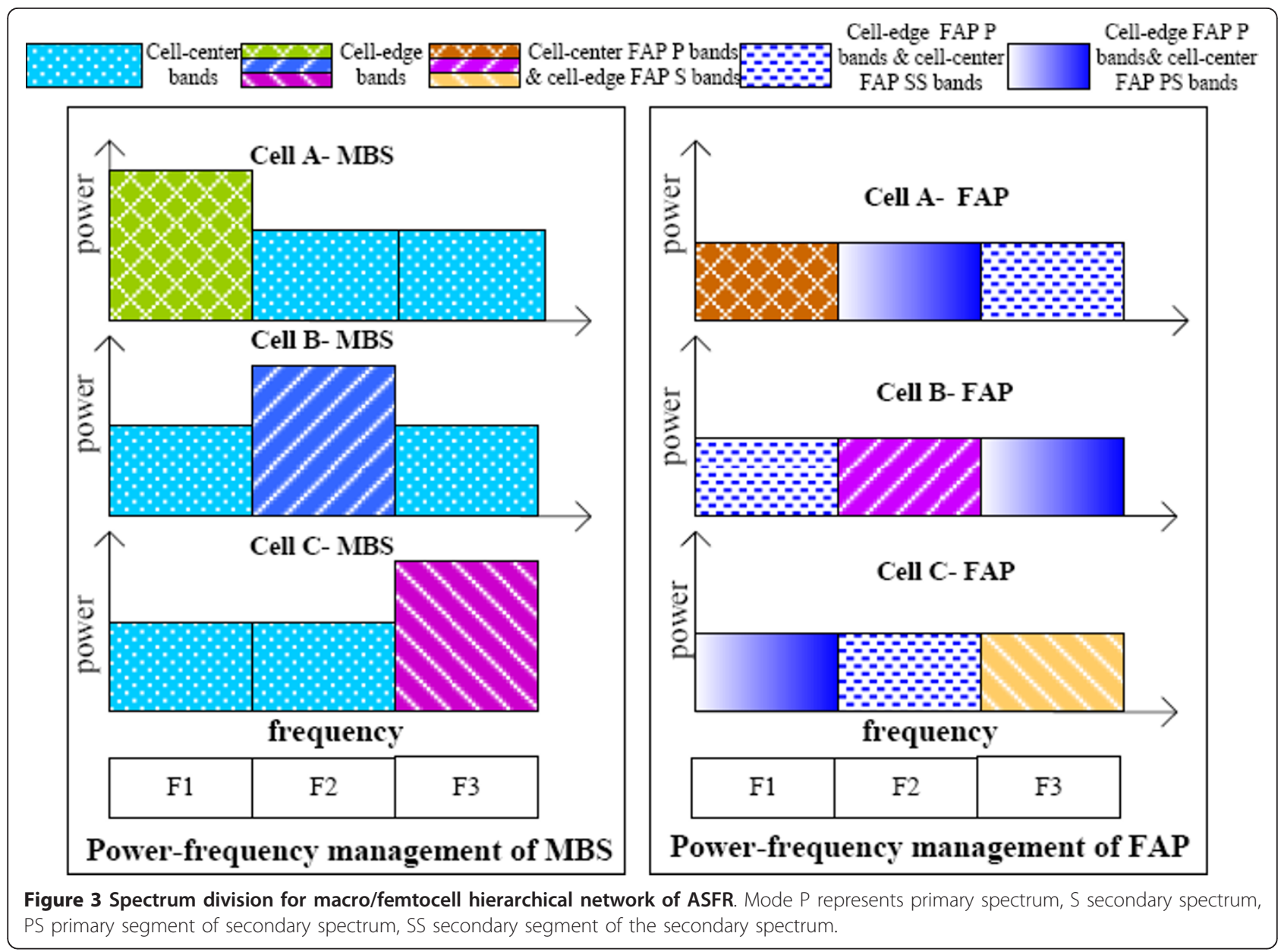

exhausted. In the following, an optimized detection criterion is generated, which further distinguish the two secondary segments of cell-edge FBSs: To improve the secondary spectrum detection efficiency, segment $(j$ $(\bmod 3)+1)$ is designed as the primary one out of the two secondary spectrum of $\mathrm{FBS}_{1, n}$, and $((j+1)(\bmod 3)$ $+1)$ as the secondary one, respectively, named primarysecondary (PS) spectrum and secondary-secondary (SS) spectrum for $\mathrm{FBS}_{1, n}$ illustrated in Figure 3. The detection of SS is triggered if and only if PS is exhausted. This means that $\mathrm{FBS}_{1, n}$ starts secondary spectrum detection from PS $(j \bmod 3+1)$ and only when the PS cannot satisfy the spectrum requirements, $\mathrm{SS}((j+1)(\bmod$ $3)+1$ ) is detected and allocated. Meanwhile, the MBS A is designed to start its spectrum allocation from a different point, i.e., SS other than PS of $\mathrm{FBS}_{1, n}$. In this way, when loading factor of MBS A is lower than $1 / 3$ and neglecting effects of other femtocells, the probability of successful secondary spectrum detection on PS of can be $100 \%$, since the PS segment of $\mathrm{FBS}_{1, n}$ is not occupied by the local macrocell. Obviously, it would be too late to trigger secondary spectrum detection after the exhaustion of the primary one. Assuming that traffic load is estimated at each cell, a mechanism is designed to support suitable detection time: (1) two thresholds are defined: load thresh 1 and load thresh 2, with load thresh 1 <load thresh 2; (2) before the load of $\mathrm{FBS}_{1, n}$ reach load thresh 1 , no channel measurements on PS is needed, and before the load of $\mathrm{FBS}_{1, n}$ reach load thresh 2, no measurements on the SS is needed. Thus, the overheads of detection are reduced and efficiency of detection is improved. Values of the two thresholds are closely correlated with the IFI condition of given environments.

\subsection{Medium-term resource management: channel and power allocation}

Through the spectrum allocation between femtocell and macrocell, the interference between two-tier networks can be mitigated, and by CR technology, the femtocells can access the macrocell's spectrum which the nearby macrocell UEs are not using, further improving the network's capacity and resource utility. However, among each femtocell of the femtocell networks, the small path 
losses may cause heavy interferences. Therefore, according to the bandwidth requirement of femtocells, the whole available spectrum including allocation and cognitive parts can be divided into several channels to be allocated to different femtocells, and the femtocells' power should be optimal configured as well.

Previous studies focused on the resource allocation between femtocells can be divided into two categories: (1) distributed self-configuring, each femtocell independently configures its work channel and power. This method has low complexity but does not consider impact on surrounding femtocells and probably causes interference. (2) Global resource allocation by calculating the optimal configuration for each femtocell. This method can achieve an optimal resource allocation but it needs a lot of information collecting and computing. In addition, this method solves the resource allocation problem by only one allocating process. In fact, the radio environment may change a lot over time as well as different femtocells switch on or off, so the real-time resource allocating is needed.

In this article, we proposed a Q-learning-based approach to deal with the resource allocation problem of femtocells for real-time. Simulation results show that the approach could configure the femtocell according to the environment, and optimize its performance without loss of other femtocells.

\subsubsection{Reinforcement-learning}

Q-learning is one kind of reinforcement-learning. The reinforcement-learning model consists of several factors [11,12]: (1) $S=\left\{s_{1}, s_{2}, \ldots, s_{n}\right\}$ denotes the finite discrete possible environment states, (2) $A=\left\{a_{1}, a_{2}, \ldots, a_{m}\right\}$ denotes the possible using actions of the agent, and (3) $r$ denotes the current reward value, (4) $\pi: S \rightarrow A$ is the agent's strategy. The relationships between these factors are shown in Figure 4:

1. Agent perceives the environment and decides the state $s$;

2. Agent choose an action $a$ according to the current strategy $\pi: S \rightarrow A$ and have an effect on the environment.

3. The environment receives the action $a$ and then transforms from state $s$ to $s$ ' by a certain probability $p$, after that it will generate a current reward $r$ and feed back to agent.

4. The agent updates its strategy $\pi: S \rightarrow A$ according to $s^{\prime}$ and $r$.

Through continuous implementation of the above process circle, the ultimate goal is to find the optimal strategies for the agent in each state $s$, making the cumulative return on a given optimization object maximum/minimum. One most common infinite horizon optimization objective is the mathematical expectation of the long-term cumulative return:

$$
V_{\pi}(s)=E\left(\sum_{t=0}^{\infty} \gamma^{t} r\left(s_{t}, a_{t}\right) \mid s_{0}=s\right)
$$

$r$ is a constant time discount factor, which reflects the importance of the future return relative to the current return, the smaller $r$ is, the less important the future return is. According to [13], (1) can be rewritten as

$$
V_{\pi}(s)=R(s, a)+\gamma \sum_{s^{\prime} \in S} P_{s, s^{\prime}}(a) V_{\pi}\left(s^{\prime}\right)
$$

$R(s, a)$ is the mathematical expectation of $r\left(s_{t}, a_{t}\right), P_{s}$, $s^{\prime}(a)$ is the probability that state $s$ transforms to $s^{\prime}$ after executing action $a$.

\subsubsection{Q-learning}

Compared with other reinforcement-learning algorithms, Q-learning has the advantages that it can directly find the optimal strategy through value iteration [14] to satisfy (2) $[15,16]$, without knowing $R(s, a)$ and $P_{s, s^{\prime}}(a)$. The specific method is each state and action pair $(s, a)$ is associated with a $\mathrm{Q}$-value $Q(s, a)$, the (2) deformation:

$$
Q_{\pi}(s, a)=R(s, a)+\gamma \sum_{s^{\prime} \in S} P_{s, s^{\prime}}(a) V_{\pi}\left(s^{\prime}\right)
$$

Its meaning is the expected cumulative return by executing action $a$ in state $s$ then following a serious of actions obeying the strategy $\pi$.

In order to obtain the optimal strategy $\pi$ that makes

$$
Q_{*}(s, a)=R(s, a)+\gamma \sum_{s^{\prime} \in S} P_{s, s^{\prime}}(a) \max _{a^{\prime} \in A} Q_{*}\left(s^{\prime}, a^{\prime}\right)
$$

where $V_{*}(s)=\max _{a \in A} Q_{*}(s, a)$,

We can make the Q-learning process as follows:

$$
Q_{t+1}(s, a)= \begin{cases}Q_{t}(s, a)+\alpha \Delta Q_{t}(s, a), & \text { if } \left.s=s_{t} \text { and } a=a_{t}\right) \\ Q_{t}(s, a), & \text { otherwise }\end{cases}
$$

where $\alpha \in[0,1)$ is the learning rate, and $\Delta Q_{t}(s, a)$ is $Q$ value update error function, as follows:

$$
\Delta Q_{t}(s, a)=r_{t}+\gamma \max _{a^{\prime} \in A} Q_{t}\left(s^{\prime}, a^{\prime}\right)-Q_{t}(s, a)
$$

It can be proved that if each $Q(s, a)$ value can be updated through an infinite number of iterations, and in this process in some appropriate way $\alpha$ gradually reduced to $0, Q(s, a)$ will converge to the optimal value of the probability of a $Q^{*}(s, a)$. At this point, the optimal strategy" $\pi$ can be obtained as

$$
\pi_{*}(s)=\underset{a \in A}{\arg \max } Q_{*}(s, a)
$$




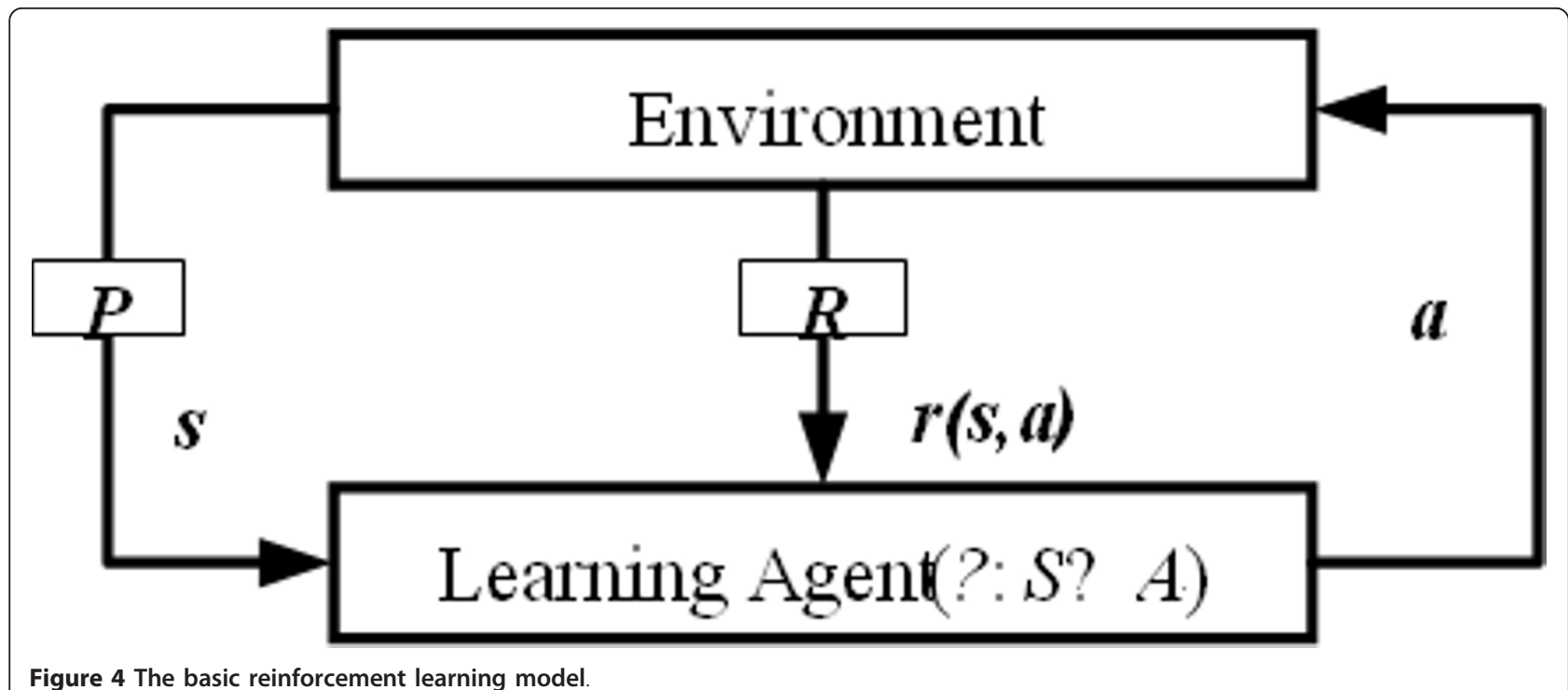

\subsubsection{Q-learning-based channel and power allocation formulation}

The proposed Q-learning-based self-configuration scheme is described as follows:

1. The FGW maintains a $Q$-value table for femtocell's self-configuration, as shown in Table 1 . This table is two-dimensional, one dimension is all possible states $s$, while the other denotes all possible actions $a$. Each unit $Q(s, a)$ denotes the $Q$ value, i.e., the value of the objective function, when the action $a$ is chosen at state $s$.

2. State $s(P, C, T)$ is mainly describing the environment of the femtocell $k$ which needs self-configuration, and we used three elements related to its neighbor femtocells to comprise $s$ : (1) $P=\left(p_{1}, \ldots, p_{N}\right)$ denotes neighbor femtocells' $(1-N)$ pilot power received by femtocell $k, p_{i} \in\{0,1,2\}$, respectively, denote low $(<10 \mathrm{dBm})$, moderate $(10-15 \mathrm{dBm})$, and high $(15-20 \mathrm{dBm})$ power. To reduce the complexity without loss of performance, the most nearest two neighbor femtocells are chosen and $N$ is supposed to be 2. (2) Neighbor femtocells' work channel vector $C=\left(c_{1} \sim c_{N}\right), c_{i} \in\{0,1,2,3\}$, respectively, denotes the four channels which the whole spectrum is divided into. (3) Neighbor femtocells' throughput vector $T=\left(t_{1} \sim t_{N}\right), t_{i} \in\{0,1,2\}$, respectively

Table 1 Q-value table for femtocell configuration

\begin{tabular}{|c|c|c|c|c|}
\hline & State & $\begin{array}{l}S_{1}\left(P_{1}, C_{1},\right. \\
\left.T_{1}\right),\end{array}$ & $\cdots$ & $\begin{array}{l}S_{\text {total }}\left(P_{\text {total }}, C_{\text {total, }}\right. \\
\left.T_{\text {total }}\right)\end{array}$ \\
\hline \multirow[t]{5}{*}{ Action } & $a_{1}\left(p_{a_{1}}, c_{a_{1}}\right)$ & $Q\left(s_{1}, a_{1}\right)$ & $\ldots$ & $Q\left(s_{\text {total, }}, a_{1}\right)$ \\
\hline & & & . & . \\
\hline & & & . & . \\
\hline & & & . & $\cdot$ \\
\hline & $a_{\max }\left(p_{a_{\max }}, c_{a_{\max }}\right)$ & $Q\left(s_{1}, a_{\max }\right)$ & $\ldots$ & $Q\left(s_{\text {total }}, a_{\max }\right)$ \\
\hline
\end{tabular}

denotes low $(<2 \mathrm{bps} / \mathrm{Hz})$, moderate $(2-5 \mathrm{bps} / \mathrm{Hz})$, and high $(>5 \mathrm{bps} / \mathrm{Hz})$ throughput.

3. Action $a\left(p_{a}, c_{a}\right)$ is the possible combinations of power $p_{k} \in\{10,15,20\} \mathrm{dBm}$ and work channel $c_{k} \in\{0,1$, $2,3\}$ that femtocell $k$ may be configured. $a\left(p_{k}, c_{k}\right) \in$ $\{0 \sim 12\}$ denotes 1 of the 12 kinds of femtocell configurations.

4. Selection criteria for action $a\left(p_{a}, c_{a}\right)$ : if we adopt the greedy algorithm, that is always at each iteration to select the action $a\left(p_{a}, c_{a}\right)$ that makes $Q(s, a)$ maximum in current state $s(P, C, T)$, probably because the initial iteration algorithm improper selection (due to lack of accumulated experience) and ultimately "cover up" the optimal strategy. In this article, we choose $a\left(p_{a}, c_{a}\right)$ more representative method: the Boltzmann distribution-based exploration algorithm. Specifically, in state $s$ $(P, C, T)$, Boltzmann distribution algorithm selected an action with following probability:

$$
p(a \mid s)=\frac{e^{Q(s, a) / T_{s}}}{\sum_{a^{\prime} \in A} e^{Q\left(s, a^{\prime}\right) / T_{s}}}
$$

where $T_{\mathrm{s}}$ is the "temperature" parameter, and decreases with the $Q$ value iterative process. Equation 8 expressed the basic idea that with the constant iteration of Q-learning algorithm update, the choice of state action will increasingly depend on the accumulated experience rather than random to explore.

5 . On reward $R(s, a)$, we consider the whole benefits of the configured femtocell $k$ and the entire network. After taking action $a\left(p_{a}, c_{a}\right)$ in state $s(P, C, T)$, femtocell $k$ obtained throughput $t_{k}$, and the throughputs of neighbor femtocell $n b_{1}$ and $n b_{2}$ change into $t_{1}{ }^{\prime}$ and $t_{2}{ }^{\prime}$ because of the interference of femtocell $k$. We define 
the reward $R(s, a)$ as follows:

$$
R(s, a)=\alpha \bullet t_{k}-\beta_{1} \bullet\left(t_{1}-t_{1}^{\prime}\right)-\beta_{2} \bullet\left(t_{2}-t_{2}^{\prime}\right)
$$

where $\alpha, \beta_{1}, \beta_{2} \in(0,1)$ are compute weights, $\alpha>\beta_{1}$ or $\beta_{2}$. Reward function means to improve the new configured femtocell $k$ 's performance as far as possible, under the premise of ensuring the gain in the overall network performance. With this reward function, the system's long-term cumulative return is the sum of network performance gains after all the resource allocations for each femtocell.

6. $Q$ value update: femtocell $k$ configures its channel and power as $a\left(p_{a}, c_{a}\right)$ in state $s(P, C, T)$, and gets its current reward $R(s, a)$. At the same time, neighbor femtocells $n b_{1}$ and $n b_{2}$ adjust their power to $p_{1}^{\prime}$ and $p_{2}^{\prime}$ according to the impact of femtocell $k$, as well as their throughput $t_{1}$ and $t_{2}$ change into $t_{1}{ }^{\prime}$ and $t_{2}{ }^{\prime}$. Therefore, the state $s(P, C, T)$ transition to $s^{\prime}(P, C, T)$, and according to $R(s, a)$ and $\max _{a^{\prime} \in A} Q_{t}\left(s^{\prime}, a^{\prime}\right)$, the $Q(s, a)$ is updated according to (5) and (6).

The Q-learning-based channel and power configuration process is shown in Figure 5, and the details are described as follows:

Initialization: $Q$ value table is cleared. To ensure that all of the state-action pairs $(s, a)$ can be fully tried, each item of the $Q$ value table is associated with a learning rate $\alpha(s, a)$ and initialized to 0 . While each state $s(P, C$, $T$ ) is associated with a temperature $T_{\mathrm{s}}$ and initialized to $T_{0}$, initialize all the visiting number $n(s, a)=0$ of each $(s, a)$. Set the time discount factor in (6) to $\gamma$.

Self-configuring trigger: There are three cases that will trigger the femtocell $k$ 's self-configuring.

1. FBS $k$ switches up;

2. $I_{k}>I_{0}, I_{0}$ denotes the set interference threshold, and $I_{k}$ is femtocell $k$ caused interference to its neighbors, which is calculated as follows:

$$
I_{k}=\sum_{i \in N B(k)} \beta_{i, k} P_{i, k}^{\text {pilot }}
$$

where $\beta_{i, k}=\left\{\begin{array}{l}1, \text { if } k \text { and } i \text { are in the same channel } \\ 0, \text { else }\end{array}, N B(k)\right.$ is the neighbor femtocell list of femtocell $k, P_{i, k}^{\text {pilot }}$ is femtocell $i$ 's received power from femtocell $k$.

3. Average SINR of femtocell $k$ 's UEs is below the threshold SINR $_{0}$ :

$$
\operatorname{SINR}_{k, \text { ave }}=\sum_{j \in U E(k)} \operatorname{SINR}_{k, j} / N=\sum_{j \in U E(k)}\left(P_{j, k}^{r} /\left(\sum_{l \neq k} P_{j, l}^{r}+n_{0}\right)\right) / N<\operatorname{SINR}_{0},
$$

$P_{j, l}^{r}$ is UE $j$ 's received power from femtocell $l, N$ is the number of femtocell $k$.

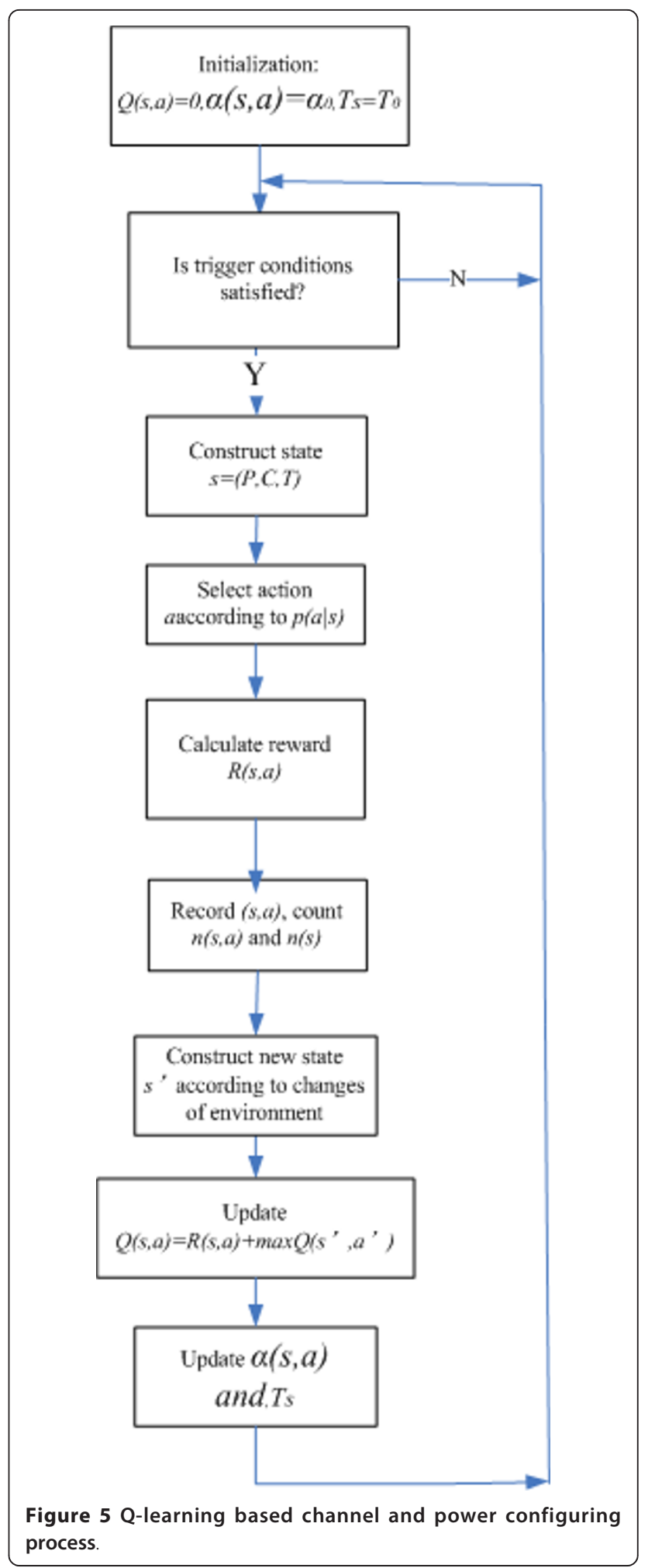

Above parameters, such as received power, interference, etc., are reported to FGW by FBSs and UEs.

Determining the state $s$ : according to the parameters reported by femtocell $k$ and its neighbor $n b_{1}$ and $n b_{2}$, 
FGW decides which states $s(P, C, T)$ femtocell $k$ is in, and finds all the $Q(s, a)$ corresponding to $s(P, C, T)$ in the $Q$ value table.

Action selection: FGW chooses an action $a\left(p_{a}, c_{a}\right)$ according to the probability calculated by (8), and configures femtocell $k$ 's channel and power, respectively, as $c_{a}$ and $p_{a}$. While recording the visited time $n(s, a)$ of $(s, a)$.

Getting reward: After the implementation of action $a$ $\left(p_{a}, c_{a}\right)$, according to the throughputs reported by the femtocells, FGW calculates $R(s, a)$ of this iteration using (9).

$Q$-value update: According to the changed throughputs and powers reported by $n b_{1}$ and $n b_{2}$, FGW decides the new state $s^{\prime}\left(P^{\prime}, C^{\prime}, T^{\prime}\right)$ which $s(P, C, T)$ transferred to after implementation of action $a\left(p_{a}, c_{a}\right)$, and updates $Q$ $(s, a)$ according to $R(s, a)$ and $\max _{a^{\prime} \in A} Q_{*}\left(s^{\prime}, a^{\prime}\right)$ using (4).

Parameters update: To ensure the convergence of strategies selection as well as $Q$-value update, we make the learning rate $\alpha(s, a)$ negative exponential declining with increasing of visited number $n(s, a)$, and temperature $T_{\mathrm{s}}$ negative exponential declining with increasing of visited number $n(s)$, where $n_{s}=\sum_{a \in A} n_{s, a}$.

\subsection{Fast resource management: coordinated multipoint transmission}

The interference between multiple femtocells can be reduced through channel and power allocation. However, due to short distance and few obstacles among femtocells, the interference may be too heavy to be well eliminated by radio resource allocation. Therefore, we considered to jointly adjust femtocells' antenna for realtime to implement fast radio resource management. In addition, large density of femtocells deployment can also bring a gain of improved joint fast resource management. Here, we only consider the downlink transmission.

In the public femtocell network, due to the lack of precise planning, there will be inevitably some coverage holes and multi-femtocell overlap area, resulting in some UEs' low received signal power or heavy interference. We plan to solve this problem through coordinated multipoint transmission technique of multiple femtocells, which in general is to make the high-gain main lobes of the femtocells toward the coverage holes and low-gain side lobes toward the overlap areas. Through collaboration of femtocells the coverage holes and interference between each other can be reduced and thus improving the network performance.

\subsubsection{Problem formulation}

In the femtocell network shown in Figure 2, suppose UE $i$ is served by femtocell $k$, then UE $i$ 's received signal SINR can be calculated as

$$
S_{k, i}=P_{k, i}^{r} /\left(\sum_{l=1, l, \neq k}^{M} P_{l, i}^{r}+n_{0}\right)=P_{k}^{t} \bullet g_{k, i}^{t} \bullet h_{k, i} /\left(\sum_{l=1, l \neq k k}^{M} \beta_{l, i}^{n} \bullet P_{l}^{t} \bullet g_{l, i}^{t} \bullet h_{l, i}+n_{0}\right)
$$

where

$h_{k, i}=10\left(-\frac{37+30 \log _{10} d_{k i}+18.3 n_{k i}\left\{\frac{n_{k i}+2}{n_{k i}+1}-0.46\right\}}{10}\right)$

$P_{k, i}^{r}$ is the received power from FBS $k$ at UE $i, P_{k}^{t}$ is the transmit power of FBS $k, g_{k, i}^{t}$ is the antenna transmit gain from FBS $k$ to UE $i, d_{k i}$ and $h_{k}, i$, respectively, denote the distance and channel gain between FBS $k$ and $\mathrm{UE} i, \beta_{l, i}^{n}$ is a binary indicator, if $\beta_{l, i}^{n}=1$, femtocell $l$ does allocate some power in channel $n$ that UE $i$ is using, zero otherwise. $\beta_{l, i}^{n}=1$ if FBS $k$ serves UE $i . n_{k i}$ denotes the number of walls in the path, $n_{0}$ denotes the effect of an interference from a MBS and additive white Gaussian noise. The total capacity of all femtocell users can be expressed as

$$
C=\sum_{i=1}^{N} C_{i}=\sum_{i=1}^{N} \log _{2}\left(1+S_{k, i}\right)
$$

Using the above formulas as a basis, we formulated an optimization problem as

$$
f_{o b j}=\max C=\max \sum_{i=1}^{N} \log _{2}\left(1+P_{k}^{t} \bullet g_{k, i}^{t} \bullet h_{k, i} / \sum_{l=1, l \neq k}^{M} \beta_{l, i}^{n} \bullet P_{l}^{t} \bullet g_{l, i}^{t} \bullet h_{l, i}+n_{0}\right)
$$

in order to maximize the network's capacity.

Previous studies about femtocell network optimizing have mainly focused on radio resource allocation schemes such as spectrum and power allocation to reduce interference and improve network capacity, i.e., selecting the optimal $\beta_{l, i}^{n}$ and $P_{l}^{t}(l=1-M, i=1-N)$. Here, we considered dynamic and real-time adjustment of femtocells' antenna gains $g_{l, i}^{t}(l=1-M)$ to give a new way of optimizing femtocell networks.

Because the movement of UE in the indoor environment is slow, during the optimization process, which is supposed to be several seconds, the $h_{k}, i, P_{k}^{t}$ are unchanged, and the objective function can be further noted as

$$
f_{\text {obj }}=\max \sum_{i=1}^{N} \log _{2}\left(1+\frac{\varepsilon_{k, i} \bullet g_{k, i}^{t}}{\sum_{l=1, l, f \neq k}^{M} \varepsilon_{l, i} \bullet g_{l, i}^{t}+n_{0}}\right)=\max \sum_{i=1}^{N} \log _{2}\left(\frac{\sum_{l=1}^{M} \varepsilon_{l, i} \bullet g_{l, i}^{t}}{\sum_{l=1, l, \neq k}^{M} \varepsilon_{l, i} \bullet g_{l, i}^{t}+n_{0}}\right)
$$

where $\varepsilon_{l, i}=\beta_{l, i}^{n} \bullet P_{l}^{t} \bullet h_{l, i}$ is a constant. Assuming matrix $\mathbf{E}, \mathbf{E}^{\prime}$, and $\mathbf{G}$, respectively, are 


$$
\begin{aligned}
& \mathbf{E}_{N, M}=\left(\begin{array}{cccc}
e_{1,1} & \ldots & \ldots & e_{1, M} \\
\cdot & \cdot & & \cdot \\
\cdot & \cdot & \cdot \\
\cdot & \cdot & \cdot & \cdot \\
e_{N, 1} & \ldots & \ldots & e_{N, M}
\end{array}\right) \text {, where } e_{n, m}=\varepsilon_{m, n}
\end{aligned}
$$

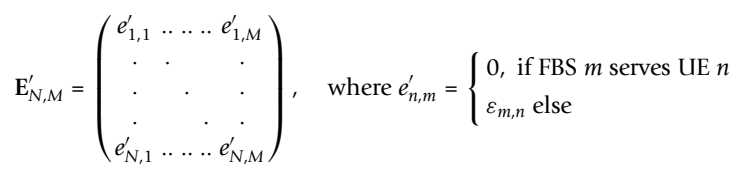

$$
\begin{aligned}
& \mathbf{G}_{M, N}=\left(g_{a, b}^{t}\right)=\left(\begin{array}{cccc}
g_{1,1}^{t} & \ldots & \cdots & g_{1, N}^{t} \\
\cdot & \cdot & & \cdot \\
\cdot & \cdot & \cdot \\
\cdot & & \cdot & \cdot \\
g_{M, 1}^{t} & \cdots & \cdots & g_{M, N}^{t}
\end{array}\right)
\end{aligned}
$$

the $m$ th row of $G_{M, N}, \tilde{g}_{m}=g_{m, y}(y=1-N)$ represented the $m$ th FBS's transmit gains to each UE. Formula (13) can be noted as

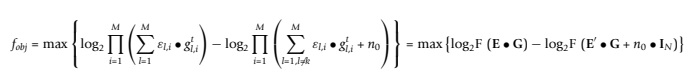

where $F\left(\mathbf{A}_{N \times N}\right)=\prod_{i=1}^{N} a_{i, i}$ and $\mathbf{I}_{N}$ is $N$ order unit matrix.

Because the $\mathbf{E}$ and $\mathbf{E}^{\prime}$ are assumed to be constant matrixes, it is clear that in order to achieve the optimal objective $f_{\text {obj }}$, we are supposed to find an optimal $G_{M, N}$ for FBSs' antenna transmit gains in different directions, which can be further noted as

$$
\mathrm{G}_{\mathrm{opt}}=\arg \max _{\mathrm{G}_{M, N}}\left\{\log _{2} F(\mathbf{E} \bullet \mathbf{G})-\log _{2} F\left(\mathbf{E}^{\prime} \bullet \mathbf{G}+n_{0} \bullet \mathbf{I}_{N}\right)\right\}
$$

\subsubsection{Coordinated antenna patterns selection}

Due to the cost and size restrictions of the FBS, the recently proposed E-plane Horns Based Reconfigurable Antenna [17,18] was used for femtocell, which is of low complexity and can form four optional patterns, one pattern can switch to arbitrary another by simple circuit switching as shown in Figure 6. Under different patterns, an FBS $k$ has different beamforming gains in each direction, i.e., different $\tilde{g}_{m}$ of $G_{M, N}$. Based on that, we proposed a coordinated multipoint transmission scheme which is to select the optimal antenna patterns combination of all FBSs, in order to obtain the approximate optimal solution of (16) with low additional complexity, which can be called as coordinated antenna patterns selecting (COPS) and noted as

$$
\begin{aligned}
& \mathrm{G}_{\mathrm{opt}}=\underset{\mathrm{G}_{M, N}}{\arg \max _{\mathrm{N}}}\left\{\log _{2} F(\mathbf{E} \bullet \mathbf{G})-\log _{2} F\left(\mathbf{E}^{\prime} \bullet \mathbf{G}+n_{0} \bullet \mathbf{I}_{N}\right)\right\} \\
& =\arg \max _{\left(\tilde{g}_{1}, \cdots \tilde{g}_{M}\right)}\left\{\log _{2} F\left(\mathbf{E} \bullet\left(\begin{array}{c}
\tilde{g}_{1} \\
\vdots \\
\tilde{g}_{M}
\end{array}\right)\right)-\log _{2} F\left(\mathbf{E}^{\prime} \bullet\left(\begin{array}{c}
\tilde{g}_{1} \\
\vdots \\
\tilde{g}_{M}
\end{array}\right)+n_{0} \bullet \mathbf{I}_{N}\right)\right\} \\
& \approx \arg \max _{\left(A P_{1}, \ldots P_{M}\right)}\left\{\log _{2} F\left(\mathrm{E} \bullet\left(\begin{array}{l}
A P_{1} \\
\vdots \\
A P_{M}
\end{array}\right)\right)-\log _{2} F\left(\mathrm{E}^{\prime} \bullet\left(\begin{array}{l}
A P_{1} \\
\vdots \\
A P_{M}
\end{array}\right)+n_{0} \bullet \mathrm{I}_{N}\right)\right\}
\end{aligned}
$$

where $A P_{k}$ denotes FBS $k$ 's antenna pattern, according to the numerical results, the COPS scheme could well improve the network capacity with a low additional complexity.

Taking into account the tradeoff between performance and complexity, we search the optimal antenna patterns combination of all FBSs using the simulated annealing algorithm (SA) rather than other heuristic-based algorithms:

$C b n_{t}=\left(A P_{1, t}, \ldots A P_{k, t}, \ldots A P_{N, t}\right)$ denotes one coordinated antenna patterns combination of all FBSs. $\mathrm{Cbn}^{*}$ is assumed as the optimal FBSs' antenna patterns combination, $f_{C b n_{t}}=\sum_{i=1}^{N} \log _{2}\left(1+S_{k, i}\right)$ is the evaluation function of the simulated annealing algorithm, and it can be calculated according to $S_{k, i}$ reported by UEs after each antenna patterns selection for FBSs. $T$ is the temperature parameter and is initiated as $T_{0}=-3 /(10 \ln 0.5)$. According to our simulation when the iteration number is more than 1000, the algorithm's performance will not be improved obviously. Therefore, max_num $=1000$ is the allowed maximized iteration number. The COPS scheme is shown in Table 2.

\section{Simulation results}

We evaluated the performance of the three proposed scheme in the two-tier (macrocell and femtocell) network shown in Figure 1 and an $M$-cell topology of an enterprise femtocell networks, as shown in Figure 2. Each femtocell has an average of $N$ randomly distributed UEs. In the simulation, continuous heterogeneous services with different weights are generated, including the full buffer, VoIP, video, HTTP, and FTP services. The system parameters are described in Table 3.

\subsection{Long-term resource management}

For the simulation of spectrum allocation between macrocell and public femtocell networks, all the users are uniformly distributed on a cell site, using the same service generation function, and the cell-center band, which is $2 / 3$ of the total available spectrum, serves approximately $2 / 3$ of the total services. Figure 7 depicts throughput performance of proposed ASFR scheme 


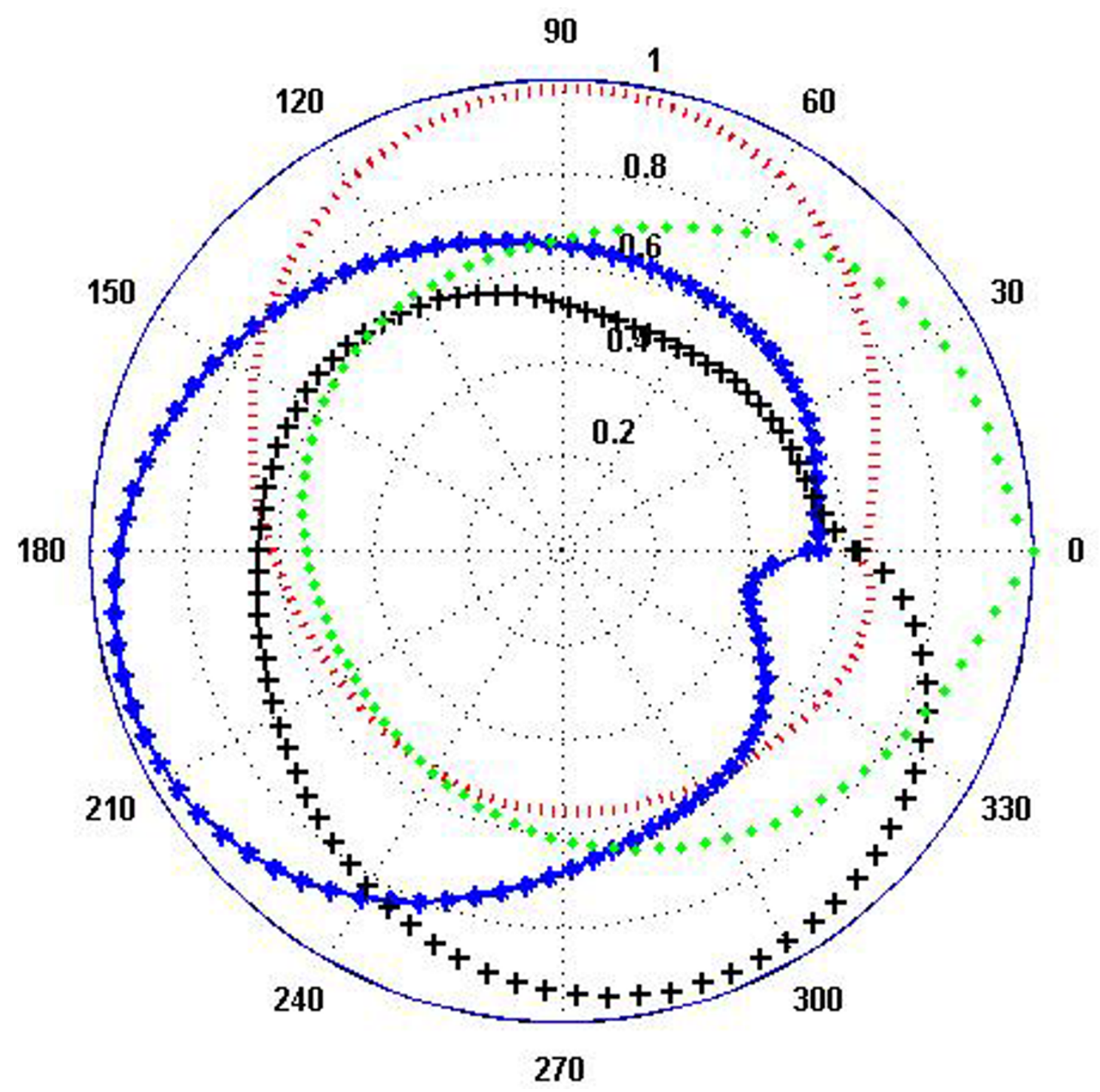

Figure 6 Four patterns of the E-plane Horns based reconfigurable antenna. The different antenna patterns can be switched to an arbitrary other one by circuit switching.

compared to a co-channel macro/femtocell setting with proportional fair scheduling. With equal priority applied to all types of service, simulation results show that, compared to the reuse one co-channel macro/femtocells, the proposed mechanism provides comparable and more stable throughput performance on both macrocells and femtocells.

\subsection{Medium-term resource management}

Based on the simulation results of the long-term resource management, we further evaluate the performance of the medium-term resource management. We simulated channel and power allocation of femtocell $k$ in a public femtocell network as shown in Figure 2, and set the user model by applying the statistical data of the UEs' distribution during the time from 8 a.m. to 8 p.m. that obtained from our institute. The indoor users moved at $1 \mathrm{~m} / \mathrm{s}$ speed in accordance with pre-set probability and routes. During the simulation, we created different scenarios in which the channels and powers of femtocell $k$ 's neighbor femtocells' are set to different values. The femtocell $k$ 's configuration of channel and power is triggered by each of the three cases mentioned in part 3. The performance parameters of all the femtocells including femtocell $k$ and its neighbors are recorded. Finally, we will evaluate the performance of proposed configuration scheme by the average values of the performance parameters. 
Table 2 Algorithm 1

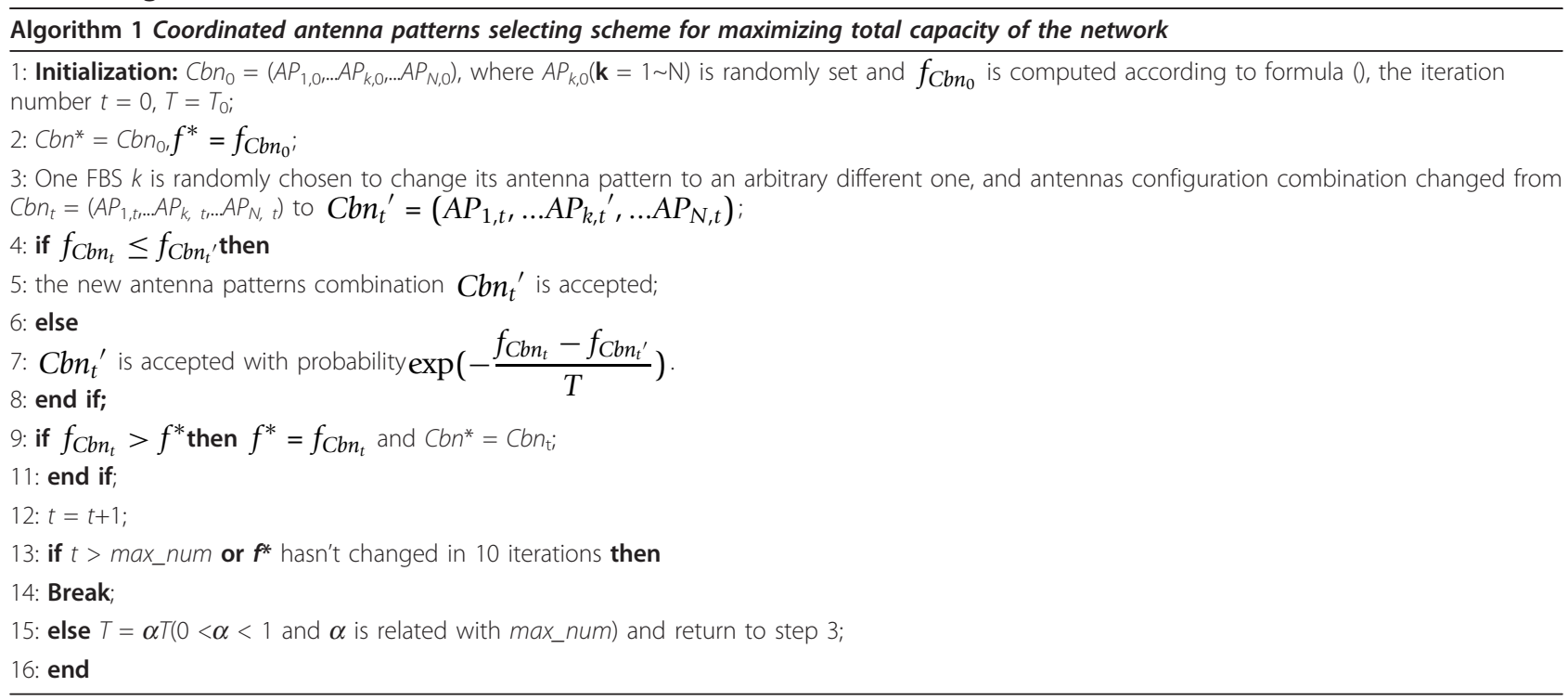

In order to better measure the performance of the proposed configuration approach, we also simulate other two configuring algorithms of channel and power: a typical distributed configuring algorithm: enhanced modified iterative water-filling [19] and a typical central configuring algorithm: joint channel allocation and power control [20]. Figures 8 and 9 depict coverage percentage and capacity of three algorithms, which are the average values of different channel allocation situations for neighbor femtocells of femtocell $k$. It can be seen from the pictures that the central configuring can achieve an excellent performance but with high complexity (which is $O\left(\left(M^{2} N+M N^{2}\right) \log _{2} N\right)$ ), the distributed configuring achieved a relatively poor performance because the femtocell which implements self-configuration does not consider the impact on neighbors. The performance of the proposed approach is very close to the central configuring but with a much lower complexity (which is $O(5 \times K), K$ is the number of actions and in our algorithm $K=12$ ).

\subsection{Fast resource management}

In addition to the simulation results of the mediumterm resource management, we further evaluate the performance of fast resource management. We implement coordinated antenna patterns selection after femtocell $k$ 's channel and power configuration in use of three different algorithms. Figures 10 and 11 show that when different medium-term resource allocation schemes reached their optimization limits, the COPS scheme can further improve the network's coverage and capacity effectively with low complexity (which is $O\left(M N \log _{2} N\right)$ ).

\section{Conclusion}

This article focused on the resource management problem in public femtocell networks. With the growing of

Table 3 Simulation parameters

\begin{tabular}{ll}
\hline Parameters & Enactment \\
\hline Macrocell/femtocell radius & $1000 \mathrm{~m} / 40 \mathrm{~m}$ \\
Average FBSs number of the macrocell & 30 \\
Average UEs number of macrocell/femtocell & $200 / 4$ \\
FBS number of the enterprise network & 9 \\
Indoor path loss & $37+30 \log 10(d)+$ Lwalls dB \\
$d$ & The distance between UE and the base station in meters. \\
Wall loss Lwalls & $15 / 10 / 7 \mathrm{~dB}$ for external/internal/light internal walls respectively, 3 dB for doors, and $1 \mathrm{~dB}$ for windows \\
Outdoor path loss & $28+35 \log 10(d) \mathrm{dB}$ \\
Receiver noise & $10 \log 10(\mathrm{k} \times \mathrm{NF} \times \mathrm{W})$ \\
Effective noise Bandwidth $W$ & $3.84 \times 106 \mathrm{~Hz}$ \\
$k$ & $1.3804 \times 1023 \times 290 \mathrm{~W} / \mathrm{Hz}$ \\
Noise figure at the UE NF & $7 \mathrm{~dB}$ \\
\hline
\end{tabular}




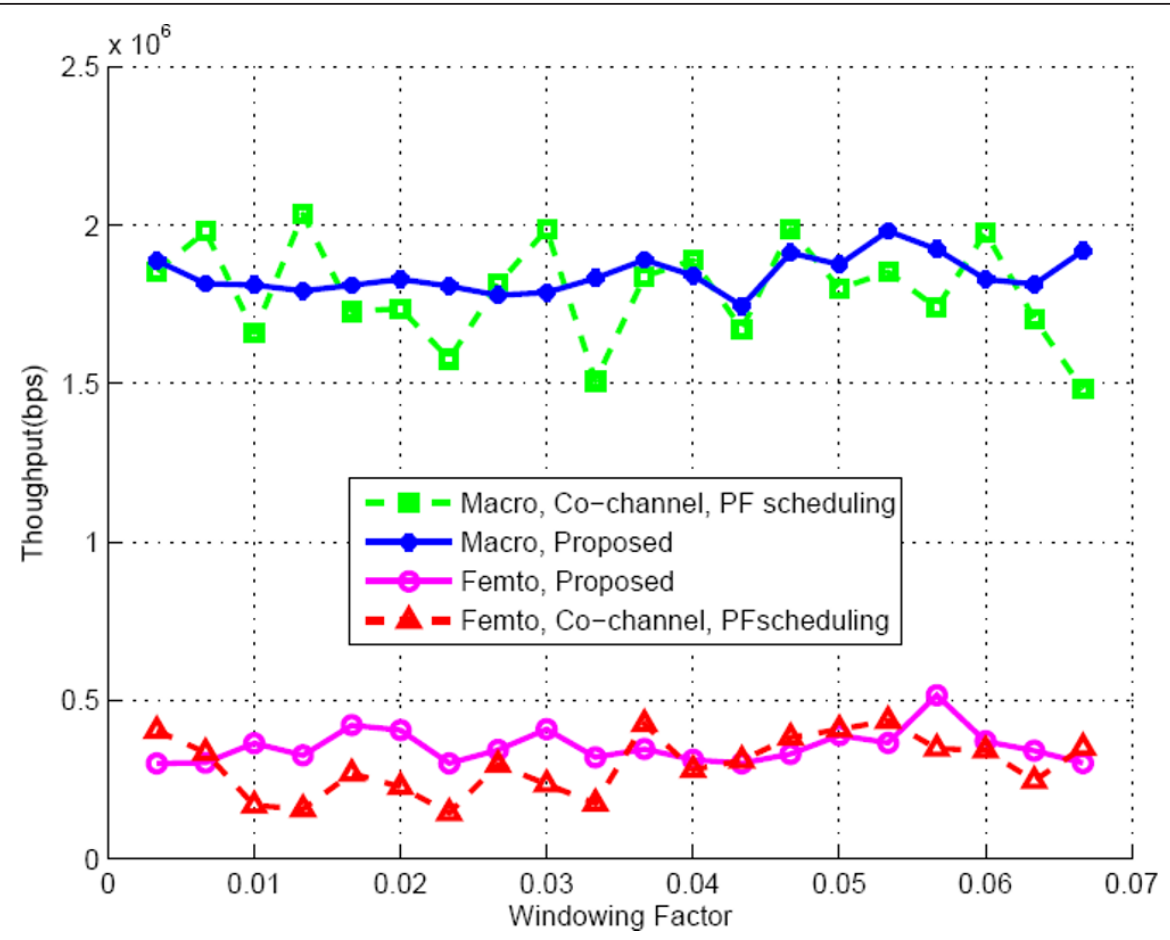

Figure 7 Indoor coverage performance. The indoor coverage percents of the target femtocell and its neighbor femtocells are calculated after resource configuring approaches with three different resource configuring approaches and are averaged in different situations in which allocated channels of neighbor femtocells are different.

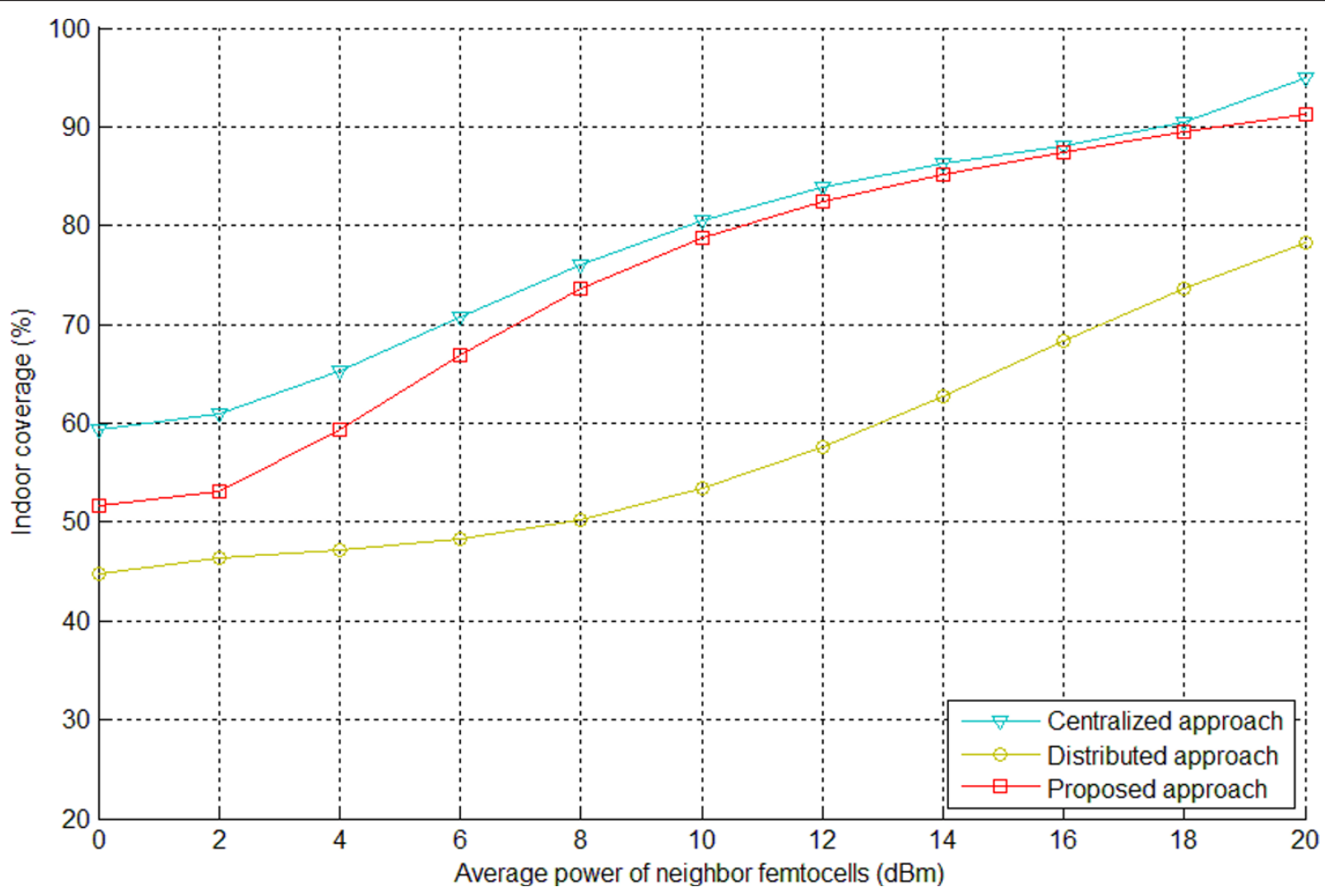

Figure 8 The throughput performance of the two-tier networks. The throughput performance of the proposed approach is compared with the co-channel partial frequency scheduling. 


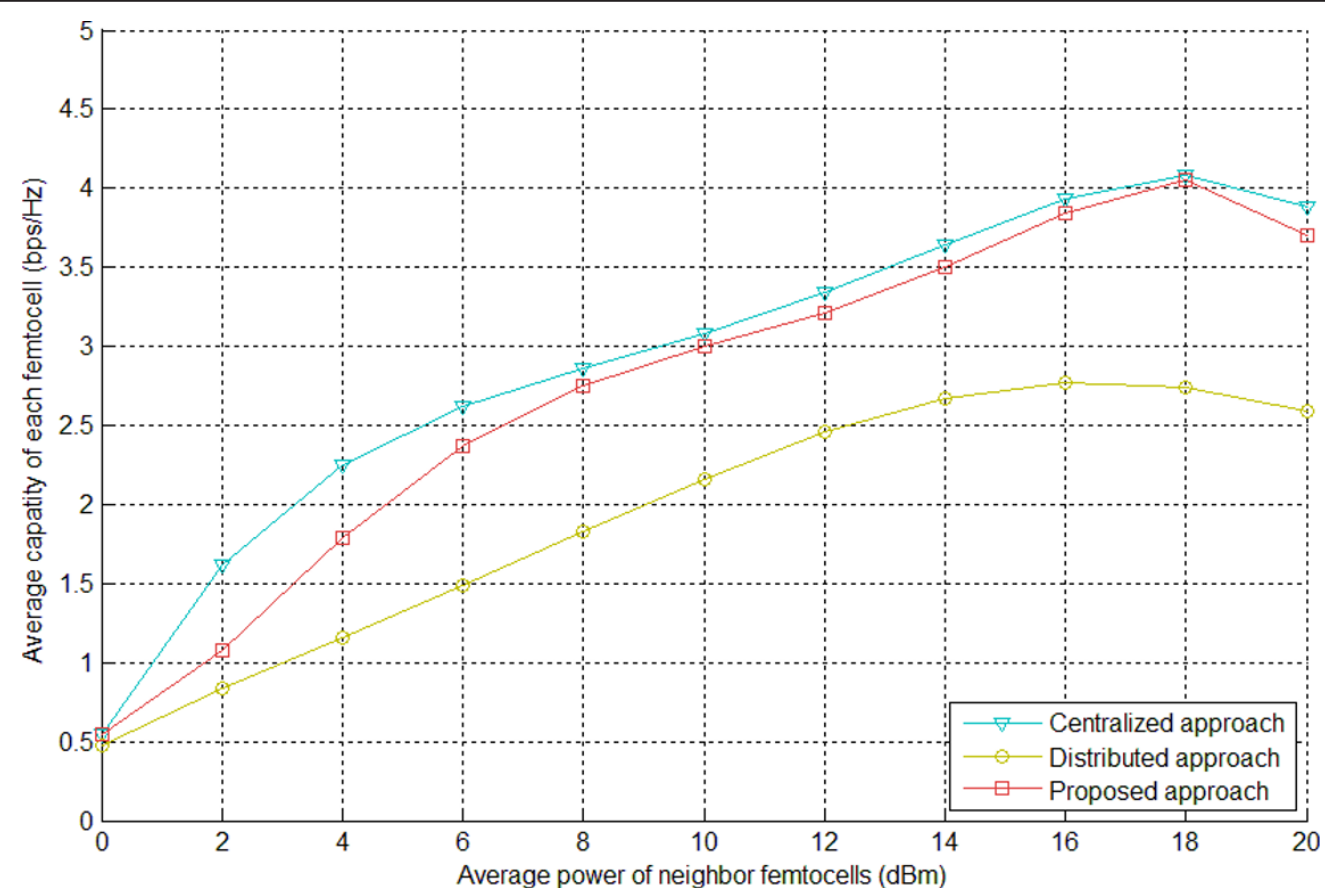

Figure 9 Average capacity of each femtocell. The average femtocell capacity is calculated after resource configuring with three different approaches and averaged in different situations in which allocated channels of neighbor femtocells are different.

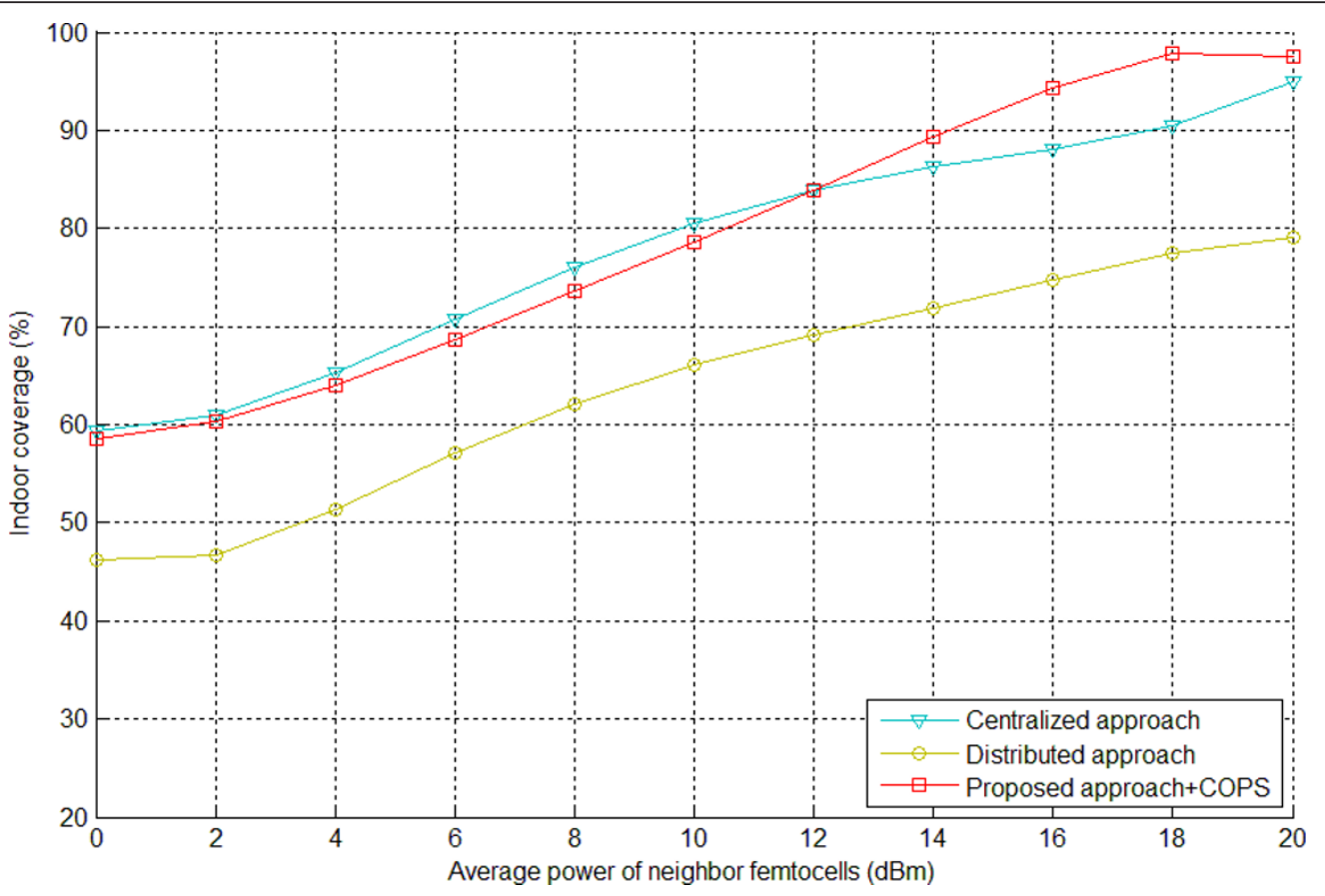

Figure 10 Indoor coverage performance after COPS. The indoor coverage performance of the target femtocell and its neighbor femtocells, after resource configuring with the proposed algorithm, is improved effectively by further implementing COPS algorithm and may exceed even the high complexity central approach. 


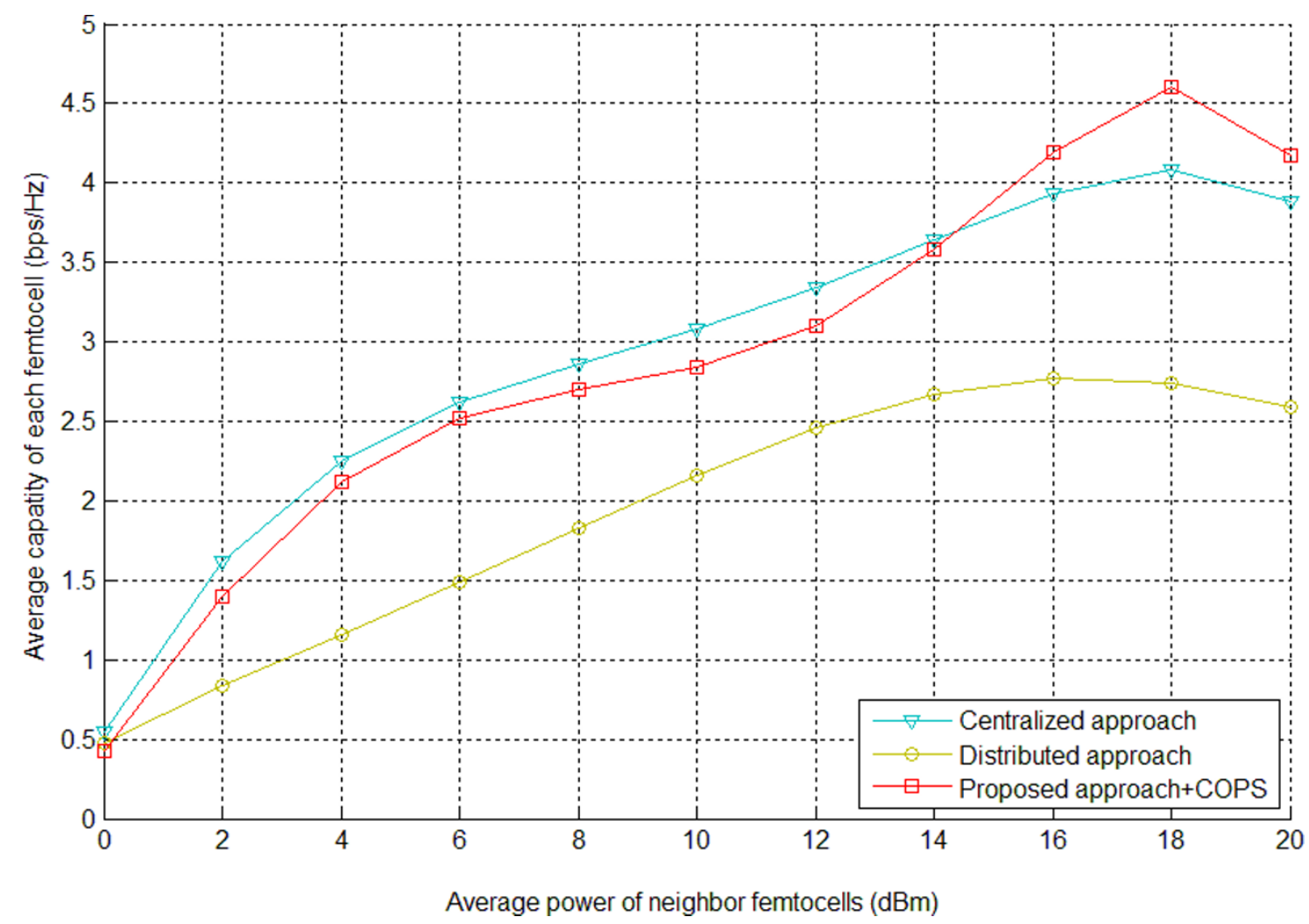

Figure 11 Average capacity of each femtocell after COPS. The average femtocell capacity after resource configuring with the proposed approach is improved effectively by further implementing COPS algorithm.

indoor communication requirement, femtocell is proposed as a solution of improving indoor coverage and network capacity. The public femtocell network is considered by operators as a promising way of meeting the increasing communication requirement of public places such as enterprise, campus, and airport. However, the deployment of public femtocell is very different from traditional macrocell or residential femtocell, as multifemtocells need to cooperate to provide coverage. Therefore, it's necessary to address the resource allocation between multiple femtocells, in order to eliminate the interference between femtocells and maximize system capacity.

In this article, we proposed a systematic approach to manage resources of femtocell network. For the longterm resource management, we adopt frequency soft reuse to solve spectrum allocation problem between macrocell and femtocell networks. In addition, we enable femtocells dynamically access spectrum allocated to macrocell through $\mathrm{CR}$, which further improves femtocell network capacity and resource utility. For the medium-term resource management, we proposed the Q-learning-based configuring approach to allocate channel and power to femtocells. At last, we proposed a coordinated antenna patterns selection scheme to implement fast resource management for femtocell networks, and make multiple femtocells to cooperate to improve the resource utility and system performance of femtocell networks.

\section{Abbreviations}

ASFR: adapted soft frequency reuse; COPS: coordinated antenna patterns selecting; CR: cognitive radio; FBS: femtocell base station; ITI: inter-tier interference; MBS: macro base station; PS: primary-secondary; SA: simulated annealing; SFR: soft frequency reuse; SOHO: small office/home office; SS: secondary-secondary; UE: user equipment.

\section{Acknowledgements}

This study was sponsored by the Key Project of National Natural Science Foundation of China (60632030, 60832009), the National Basic Research Program of China (973 Program) (2009CB320406), the National Key Technology R\&D Program of China (2010ZX03003-001-01) and the Doctoral Fund of Ministry of Education of China (20070013017) and the contributions of the colleagues from Wireless Technology Innovation Institute of Beijing University of Posts and Telecommunications are hereby acknowledged.

\section{Competing interests}

The authors declare that they have no competing interests.

Received: 1 July 2011 Accepted: 23 November 2011 Published: 23 November 2011 


\section{References}

1. V Chandrasekhar, JG Andrews, A Gatherer, Femtocell networks: a survey. IEEE Commun Mag. 46(9), 59-67 (2008)

2. Presentation by ABI Research, Picochip, Airvana, IP access, Gartner, Telefonica Espana. 2nd International Conference Home Access Points and Femtocells, http://www.avrenevents.com/dallasfemto2007/

3. SR Saunders, S Carlaw, A Giustina, RR Bhat, VS Rao, R Siegberg, Femtocells: Opportunities and Challenges for Business and Technology (Wiley, 2009). ISBN: 978-0-470-74816-9

4. I Ashraf, H Claussen, LTW Ho, Distributed radio coverage optimization in enterprise femtocell networks, in IEEE ICC 2010 proceedings

5. S Choi, T Lee, M Chung, H Choo, Adaptive coverage adjustment for femtocell management in a residential scenario. Management Enabling the Future Internet for Changing Business and New Computing Services, 221-230 (2009)

6. H Claussen, L Ho, L Samuel, Self-optimization of coverage for femtocell deployments, in Proceedings of Wireless Telecommunication Symposium, pp. 278-285

7. H Claussen, F Pivit, Femtocell coverage optimization using switched multielement antennas, in IEEE International Conference on Communication, pp. 1-6 (2009)

8. A Duran, G Carrasco, UMTS femtocell performance in massive deployments: capacity and GoS implications. Bell Labs Tech J. 14(2), 185-202 (2009). doi:10.1002/bltj.20379

9. V Chandrasekhar, JG Andrews, Uplink capacity and interference avoidance for two-tier cellular networks, in Proceedings of IEEE Global Telecommunication Conference (GLOBECOM), Washington, DC, 2007, pp 3322-3326

10. X Zheng, B Walke, Frequency reuse techniques for attaining both coverage and high spectral efficiency in ofdma cellular systems, in WCNC 2010

11. R Sutton, A Barto, Reinforcement Learning: An Introduction (MIT Press, 1998)

12. N Samaan, A Karmouch, An automated policy-based management framework for differentiated communication systems. IEEE J Sel Areas Commun. 23(12), 2236-2247 (2005)

13. CJCH Watkins, Learning from delayed rewards, Ph.D. Dissertation (Cambridge University, Cambridge, UK, 1989)

14. LP Kaelbling, ML Littman, X Wang, et al, Reinforcement learning: a survey. J Artif Intell Res. 4, 237-285 (1996)

15. CJCH Watkins, P Dayan, Q-learning. Mach Learn. 8, 279-292 (1992)

16. J Nie, S Haykin, A Q-learning-based dynamic channel assignment technique for mobile communication systems. IEEE Trans Veh Technol. 48(5), 1676-1687 (1999). doi:10.1109/25.790549

17. A Tsai, J Huang, L Wang, R Hwang, High capacity femtocells with directional antennas, in IEEE WCNC 2010 proceedings

18. H Claussen, F Pivit, Femtocell coverage optimization using switched multielement antennas, in ICC 2009

19. K Lee, O Jo, D Cho, Cooperative resource allocation for guaranteeing intercell fairness in femtocell networks. IEEE Commun Lett. 15(2), 222-235 (2011)

20. S Guruacharya, D Niyato, E Hossain, D Kim, Hierarchical competition in femtocell-based cellular networks, in IEEE Globecom 2010 proceedings

doi:10.1186/1687-1499-2011-181

Cite this article as: Li et al:: Radio resource management for public femtocell networks. EURASIP Journal on Wireless Communications and Networking 2011 2011:181.

\section{Submit your manuscript to a SpringerOpen ${ }^{\mathcal{O}}$ journal and benefit from:}

- Convenient online submission

- Rigorous peer review

- Immediate publication on acceptance

- Open access: articles freely available online

- High visibility within the field

- Retaining the copyright to your article

Submit your next manuscript at $\gg$ springeropen.com 